\title{
Expressions for precession consistent with the IAU 2000A model
}

\section{Considerations about the ecliptic and the Earth Orientation Parameters}

\author{
P. Bretagnon ${ }^{\dagger}$, A. Fienga, and J.-L. Simon \\ Institut de Mécanique Céleste, Observatoire de Paris, UMR 8028 du CNRS, 77 avenue Denfert-Rochereau, 75014 Paris, France
}

Received 3 June 2002 /Accepted 29 November 2002

\begin{abstract}
Since the adoption by the XXIVth General Assembly of an accurate model of nutation, the IAU encourages the development of new expressions for precession consistent with the new model. This paper presents new expressions for the precession quantities issued from the analytical solution of the rotation of the rigid Earth SMART97 (Bretagnon et al. 1998) which provides together the precession and the nutation. These expressions include the new value of the precession rate of the equator in longitude. As the SMART97 series are close to the Souchay et al. (1999) series used to build the new model, our expressions are consistent with the IAU 2000 Precession-Nutation Model. In the other parts of the paper, we discuss some concepts. In Sect. 3, we propose the definition of a conventional ecliptic plane close to the mean ecliptic J2000 and with a non-rotating origin. Section 4 deals with the Earth Orientation Parameters. We show that the celestial pole offsets, as well as the polar motion can be described with the Euler's angles. At last, in Sect. 5, we give some recommendations about the precession-nutation variables and the arguments of the series of the nutation.
\end{abstract}

Key words. celestial mechanics - Earth

\section{Introduction}

The resolution B1.6 adopted at the XXIVth General Assembly of the IAU (IAU 2000) recommends that, beginning on 1 January 2003, the IAU 1976 Precession Model and IAU Theory of Nutation be replaced by the precession-nutation model IAU 2000A (or its shorter version IAU 2000B). These models are issued from the model MHB2000, based on the transfer function of Mathews et al. (2002) applied to the nutation series of Souchay et al. (1999).

These nutation series do not provide expressions for precession and for this reason the IAU "encourages the development of new expressions for precession consistent with the IAU 2000A model" (encouragement 3 of resolution B1.6). Meanwhile, the IERS Conventions 2000 (McCarthy 2002) recommend the use of the precessional formulae derived from Lieske et al. (1977) with improved numerical values for the precession rate of the equator in longitude and obliquity. These formulae are based upon the use of the secular variations of the ecliptic pole from Newcomb's theory of the Sun and of old value of the precession constant and of the masses of the planets. Up to date developments have been computed by Simon et al. (1994) and Williams (1994) but they are not really issued from a new theory of the precession-nutation.

Send offprint requests to: J.-L. Simon, e-mail: simon@imcce. fr

$\dagger$ Pierre Bretagnon, died on November 17, 2002.
In this paper we present, at first, new expressions for the precession quantities, issued from the analytical solution of the rigid Earth SMART97 (Bretagnon et al. 1998) which provides together developments for the precession and series for the nutation. These expressions are consistent with the IAU 2000A model and are more precise than the ones given by Lieske et al. In a second part, we propose and discuss the concept of a conventional ecliptic. In a third part, we introduce some ideas concerning the Earth Orientation Parameters (EOP) in connection with the Euler's angles. At last, we give some considerations on the precession-nutation variables and on the arguments of the series of the nutation.

\section{New expressions for the precession quantities}

\subsection{The precession quantities}

The precession quantities are illustrated in Fig. 1. $\gamma_{J 2000}$ is the equinox J2000, $\gamma_{\mathrm{D}}$ the equinox of date, $N$ the ascending node of the ecliptic of date on the ecliptic $\mathrm{J} 2000$ and $R$ the intersection of the equator of date and the ecliptic J2000. The notations are those defined by Lieske et al.

\subsection{The solution SMART97}

SMART97 gives analytical solutions for each of the three Euler's angles $\psi, \omega, \varphi$. The Euler's angles are reckoned positively in positive rotation, in contrast to lunisolar (and general) 


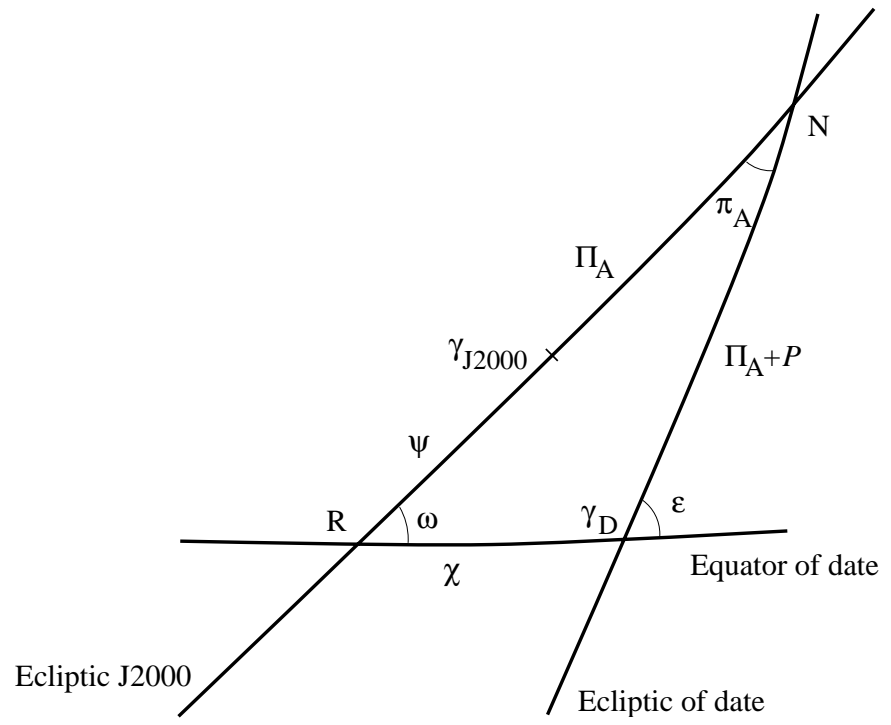

Fig. 1. Ecliptic and equator J2000 and of date.

precession. From the solutions for the Euler's angles, we deduce the solutions for the variables $\mathcal{P}$ and $\varepsilon$. All these solutions combine precession polynomials and nutation series.

\subsection{Notations for the precession and nutation parts of the variables $\mathcal{P}, \psi, \epsilon$ and $\omega$}

Table 1 gives the notations used in this paper and by the IAU for the precession and nutation parts of the variables $\mathcal{P}, \psi, \epsilon$ and $\omega$. The differences come from the convention we use for the angles (angles reckoned positively in positive rotation) and from the adoption of notations more convenient than those used by the IAU for $\Delta \mathcal{P}, \Delta \psi$ and $\Delta \omega$.

\subsection{The values of the precession rates and of the obliquity at J2000.0}

The IERS Conventions 2000 recommends to associate the Lieske et al. expressions with improved numerical values for the precession rate of the equator in longitude and obliquity, and for the obliquity at J2000.0. With our notations, these corrections are

$\delta\left(-\psi_{A}\right)=-2.9965^{\prime \prime} / \mathrm{tjy}$

$\delta\left(-\omega_{A}\right)=-0.2524^{\prime \prime} / \mathrm{tjy}$

$\varepsilon_{0}=84381.448^{\prime \prime}=23^{\circ} 26^{\prime} 21.448^{\prime \prime}$

where tjy means one thousand Julian years.

The value of $\delta \psi_{A}$ corresponds, for the precession rate of the equator in longitude, to the value

$\frac{\mathrm{d}\left(-\psi_{A}\right)}{\mathrm{d} t}=50384.7875^{\prime \prime} / \mathrm{tjy}$.

This value is slightly different from the result from SMART97 (50384.56488"/tjy) but it can be regarded as deduced from observations. The difference to the value of SMART97 can be explained by a change of the constant of the general precession. In SMART97 the William's value (1994), 50 287.7"/tjy, was
Table 1. Notations used in this paper and by the IAU for the precession and nutation parts of the variables $\mathcal{P}, \psi, \varepsilon$ and $\omega$.

\begin{tabular}{rrrr}
\hline \hline \multicolumn{3}{c}{ This paper } & \multicolumn{2}{c}{ IAU } \\
\hline Precession & Nutation & Precession & Nutation \\
\hline $\mathcal{P}_{\mathcal{A}}$ & $\Delta \mathcal{P}$ & $\mathcal{P}_{\mathcal{A}}$ & $\Delta \psi$ \\
$\psi_{A}$ & $\Delta \psi$ & $-\psi_{A}$ & $-\Delta \psi_{1}$ \\
$\varepsilon_{A}$ & $\Delta \varepsilon$ & $\varepsilon_{A}$ & $\Delta \varepsilon$ \\
$\omega_{A}$ & $\Delta \omega$ & $-\omega_{A}$ & $-\Delta \varepsilon_{1}$ \\
\hline
\end{tabular}

used. The value of this constant consistent with the improved value of $\mathrm{d} \psi_{A} / \mathrm{d} t$ given by the IERS is

$\mathcal{P}_{1}=50287.92262^{\prime \prime} / \mathrm{tjy}$.

Finally, in this paper, we use the values (2) and (3).

On the contrary, the value of the precession rate of the equator in obliquity is given by the theory and must not be given by the observations. Unfortunately, as pointed by Williams (1994), the Lieske et al. formulae as well as the ones given by Simon et al. include an error and give zero for this value. This error justifies the correction recommended by the IERS. Here, we give the result coming from SMART97

$\frac{\mathrm{d}\left(-\omega_{A}\right)}{\mathrm{d} t}=-0.26501^{\prime \prime} / \mathrm{tjy}$.

At last the value of $\varepsilon_{0}$ recommended by the IERS is the IAU 1976 value; in this paper we use

$\varepsilon_{0}=-\omega_{0}=84381.40880^{\prime \prime}=23^{\circ} 26^{\prime} 21.40880^{\prime \prime}$.

This point will be discussed in Sect. 3 .

\subsection{Precession formulae from SMART97}

As indicated in Sect. 2.2, the solution SMART97 gives together the precession and the nutation. The precession quantities are the secular parts of the developments of the variables. Table 2 gives the secular parts $P_{A}, Q_{A}, \pi_{A}, \Pi_{A}, \mathcal{P}_{A}, \theta_{A}, \zeta_{A}, z_{A}, \varepsilon_{A},-\omega_{A}$, $-\psi_{A}$ and $\chi_{A}$ of the variables $P=\sin \pi \sin \Pi, Q=\sin \pi \cos \Pi$, $\pi, \Pi, \mathcal{P}, \theta, \zeta, z, \varepsilon,-\omega,-\psi, \chi$. These polynomials are an improvement of the polynomials given in Bretagnon et al. (1997). Moreover, they include the changes due to the new value of the precession constant according to the derivative formulae of Simon et al.

The expression for Greenwich Mean Sidereal Time (GMST) at $0^{\mathrm{h}}$, coming from $\int \cos \omega_{A} \frac{\mathrm{d} \psi_{A}}{\mathrm{~d} t} \mathrm{~d} t-\chi_{A}$ is

$$
\begin{aligned}
\operatorname{GMST}\left(0^{\mathrm{h}}\right)= & 24110^{\mathrm{s}} .54841+86401847.942217 t \\
& +9.320608 t^{2}-0.000189 t^{3} \\
& -0.019697 t^{4}-0.000097 t^{5} \\
& +0.000017 t^{\mathrm{s}}+0^{\mathrm{s}} .000001 t^{7}
\end{aligned}
$$

where $t$ is in tjy from $\mathbf{J} 2000$. The change in the secular part of GMST is essentially due to the correction in the precession rate of the constant of the general precession (see also Williams 1994). 
Table 2. Secular parts of the precession quantities issued from SMART97 (Bretagnon et al. 1998). Unit is arcsec. Time $t$ is in thousands of Julian years (tjy) from J2000.

\begin{tabular}{rrrrrrrrr}
\hline \hline & $t^{0}$ & \multicolumn{1}{c}{$t$} & $t^{2}$ & $t^{3}$ & $t^{4}$ & $t^{5}$ & $t^{6}$ & $t^{7}$ \\
\hline$P_{A}$ & 0.00000 & 41.99604 & 19.39715 & -0.22350 & -0.01035 & 0.00019 & 0.0 & 0.0 \\
$Q_{A}$ & 0.00000 & -468.09550 & 5.10421 & 0.52228 & -0.00569 & -0.00014 & 0.00001 & 0.0 \\
$\pi_{A}$ & 0.00000 & 469.97560 & -3.35050 & -0.12370 & 0.00030 & 0.0 & 0.0 & 0.0 \\
$\Pi_{A}$ & 629543.988 & -8679.218 & 15.342 & 0.005 & -0.037 & -0.001 & 0.0 & 0.0 \\
$\mathcal{P}_{\mathcal{A}}$ & 0.00000 & 50287.92262 & 111.24406 & 0.07699 & -0.23479 & -0.00178 & 0.00018 & 0.00001 \\
$\theta_{A}$ & 0.00000 & 20041.90936 & -42.66980 & -41.82364 & -0.07291 & -0.01127 & 0.00036 & 0.00009 \\
$\zeta_{A}$ & 2.72767 & 23060.80472 & 30.23262 & 18.01752 & -0.05708 & -0.03040 & -0.00013 & 0.0 \\
$z_{A}$ & -2.72767 & 23060.76070 & 109.56768 & 18.26676 & -0.28276 & -0.02486 & -0.00005 & 0.0 \\
$\varepsilon_{A}$ & 84381.40880 & -468.36051 & -0.01667 & 1.99911 & -0.00523 & -0.00248 & -0.00003 & 0.0 \\
$-\omega_{A}$ & 84381.40880 & -0.26501 & 5.12769 & -7.72723 & -0.00492 & 0.03329 & -0.00031 & -0.00006 \\
$-\psi_{A}$ & 0.00000 & 50384.78750 & -107.19530 & -1.14366 & 1.32832 & -0.00940 & -0.00350 & 0.00017 \\
$\chi_{A}$ & 0.00000 & 105.57686 & -238.13769 & -1.21258 & 1.70238 & -0.00770 & -0.00399 & 0.00016 \\
\hline
\end{tabular}

Table 3. Differences between Table 2 and Lieske et al. (1977) improved by (1) (McCarthy 2002) and evaluation of the difference over 100 yrs. Unit is arcsec. Time $t$ is in thousands of Julian years (tjy) from $\mathbf{J} 2000$.

\begin{tabular}{rrrrrrrrrrr}
\hline \hline & \multicolumn{2}{c}{$t^{0}$} & \multicolumn{1}{c}{$t$} & $t^{2}$ & $t^{3}$ & $t^{4}$ & $t^{5}$ & $t^{6}$ & $t^{7}$ & $\begin{array}{c}\text { Difference } \\
\text { over 100 yrs }\end{array}$ \\
\hline$P_{A}$ & 0.00000 & 0.02004 & -0.04985 & -0.0445 & -0.01035 & 0.00019 & 0.0 & 0.0 & 0.003 \\
$Q_{A}$ & 0.00000 & 0.05450 & 0.04521 & 0.17828 & -0.00569 & -0.00014 & 0.00001 & 0.0 & 0.006 \\
$\pi_{A}$ & 0.00000 & -0.05340 & -0.04850 & -0.18370 & 0.00030 & 0.0 & 0.0 & 0.0 & 0.006 \\
$\Pi_{A}$ & -10.994 & 18.871 & 11.806 & 0.005 & -0.037 & -0.001 & 0.0 & 0.0 & 0.006 \\
$\mathcal{P}_{\mathcal{A}}$ & 0.00000 & -0.04688 & 0.13106 & 0.08299 & -0.23479 & -0.00178 & 0.00018 & 0.00001 & 0.006 \\
$\theta_{A}$ & 0.00000 & -1.19964 & -0.00480 & 0.00936 & -0.07291 & -0.01127 & 0.00036 & 0.00009 & 0.120 \\
$\zeta_{A}$ & 2.72767 & -1.37628 & 0.04462 & 0.01952 & -0.05708 & -0.03040 & -0.00013 & 0.0 & 0.138 \\
$z_{A}$ & -2.72767 & -1.42030 & 0.09968 & 0.06376 & -0.28276 & -0.02486 & -0.00005 & 0.0 & 0.143 \\
$\varepsilon_{A}$ & -0.03920 & -0.21051 & 0.04233 & 0.18611 & -0.00523 & -0.00248 & -0.00003 & 0.0 & 0.021 \\
$-\omega_{A}$ & -0.03920 & -0.01261 & 0.00069 & -0.00123 & -0.00492 & 0.03329 & -0.00031 & -0.00006 & 0.001 \\
$-\psi_{A}$ & 0.00000 & 0.00000 & 0.06370 & 0.00334 & 1.32832 & -0.00940 & -0.00350 & 0.00017 & 0.001 \\
$\chi_{A}$ & 0.00000 & 0.05086 & -0.07369 & -0.08758 & 1.70238 & -0.00770 & -0.00399 & 0.00016 & 0.006 \\
\hline
\end{tabular}

The polynomial part of the third Euler's angle $\varphi$ which measures the diurnal rotation of the Earth, coming from the relation $\varphi=\mathrm{GMST}+\chi$, is

$\varphi=4.89496121282+2301216.753652525 t$

$-0.000476710 t^{2}-0.000005892 t^{3}+0.000006821 t^{4}$

$-0.000000044 t^{5}-0.000000018 t^{6}+0.000000001 t^{7}$

where the unit is radian and time $t$ is in tjy. Note that the constant part and the linear part of $\varphi$ are integration constants.

\subsection{Comparison with the formulae of Lieske et al. (1977) improved by McCarthy (2002)}

Table 3 gives the differences between our expressions and the Lieske et al. ones improved by the values of $\delta \psi_{A}$ and $\delta \omega_{A}$ given by (1). The last column of the table gives the maximum difference, for each variable, between the value given by our polynomials and the value given by the polynomials of Lieske et al. over 100 years. We see that those differences are important and superior to the precision of the low-precision model IAU 2000B. Note that the terms in $t^{4}$ which do not exist in the expressions of Lieske et al. can reach, over $100 \mathrm{yrs}$, more than $100 \mu$ as for some variables $(\psi, \chi)$ and must be included in the model IAU 2000A.

\subsection{Consistence with the IAU Model of the nutation}

The accuracy of SMART97 is about $2 \mu$ as for $\psi$ and $\varphi$ and $0.6 \mu$ as for $\omega$, over 1970-2020. It is the best available solution of the rigid Earth rotation. But, at the present time, the implementation of the geophysical contributions by convolution with the Earth's transfer functions of Mathews has not yet been provided. So the new IAU model is based on the nutation series of Souchay et al. The differences between our nutation series and the Souchay et al. series are less than $200 \mu$ as. They are considerably smaller than the differences between the Souchay 
series and the Kinoshita series (1977), which from is the IAU 1980 model, which reach several $0.001^{\prime \prime}$. So our formulae must be regarded as consistent with the new IAU model.

\subsection{Derivatives of the precession quantities with respect to the constants $\mathcal{P}_{1}$ and $\varepsilon_{0}$}

From the formulae of Simon et al., we can compute the corrections $\Delta X$ of the precession quantities $\mathrm{X}$ given by future improvements $\Delta \mathcal{P}_{1}$ and $\Delta \varepsilon_{0}$ of $\mathcal{P}_{1}$ and $\varepsilon_{0}$. These corrections have the form

$\Delta X=\frac{\partial X}{\partial \mathcal{P}_{1}} \Delta \mathcal{P}_{1}+\frac{\partial X}{\partial \varepsilon_{0}} \Delta \varepsilon_{0}$.

For all the precession quantities of Table 1, these corrections to be added to the polynomials are given by

$$
\begin{aligned}
\Delta P_{A}= & 0 \\
\Delta Q_{A}= & 0 \\
\Delta \pi_{A}= & 0 \\
\Delta \Pi_{A}= & 0 \\
\Delta \mathcal{P}_{A}= & \Delta \mathcal{P}_{1} t+\left(0.0031 \Delta \mathcal{P}_{1}\right) t^{2} \\
\Delta \theta_{A}= & \left(0.3978 \Delta \mathcal{P}_{1}+0.2236 \Delta \varepsilon_{0}\right) t \\
& -\left(0.0008 \Delta \mathcal{P}_{1}\right) t^{2}-\left(0.0025 \Delta \mathcal{P}_{1}\right) t^{3} \\
\Delta \zeta_{A}= & \left(0.4588 \Delta \mathcal{P}_{1}-0.0485 \Delta \varepsilon_{0}\right) t \\
& +\left(0.0009 \Delta \mathcal{P}_{1}\right) t^{2}+\left(0.0011 \Delta \mathcal{P}_{1}\right) t^{3} \\
\Delta z_{A}= & \left(0.4588 \Delta \mathcal{P}_{1}-0.0485 \Delta \varepsilon_{0}\right) t \\
& +\left(0.0028 \Delta \mathcal{P}_{1}\right) t^{2}+\left(0.0011 \Delta \mathcal{P}_{1}\right) t^{3} \\
\Delta \varepsilon_{A}= & \Delta \varepsilon_{0} \\
\Delta \omega_{A}= & \Delta \varepsilon_{0}-\left(0.0003 \Delta \mathcal{P}_{1}\right) t^{3} \\
\Delta \psi_{A}= & \left(\Delta \mathcal{P}_{1}-0.0013 \Delta \varepsilon_{0}\right) t+\left(-0.0021 \Delta \mathcal{P}_{1}+0.0021 \Delta \varepsilon_{0}\right) t^{2} \\
\delta \chi_{A}= & \left(-0.0012 \Delta \varepsilon_{0}\right) t+\left(-0.0057 \Delta \mathcal{P}_{1}+0.0027 \Delta \varepsilon_{0}\right) t^{2}
\end{aligned}
$$

where $\Delta \mathcal{P}_{1}$ is measured in arcseconds by thousands of years and $\Delta \varepsilon_{0}$ in arcseconds.

\section{Definition of a conventional ecliptic}

ICRF was adopted in 1997 by the Recommendation B2 of the XXIIIrd IAU General Assembly (IAU 1997). It is a reference frame defined by a group of extragalactic sources. It is independent of the equator and the equinox but was selected close to the equator J2000. One notes that the mean ecliptic J2000 is linked to the ICRF by two rotations depending on the solution, numerical or analytical, of the motion of the Earth. From the last analytical solution built at the IMCCE, we have

1) a rotation about the $z$ axis of $\varphi$ with

$\varphi=-0.053727^{\prime \prime}=-0.000000260476 \mathrm{rad}$

2) a rotation about the $x$ axis of $\varepsilon$ with

$\varepsilon=23^{\circ} 26^{\prime} 21.408800^{\prime \prime}=0.409092614174 \mathrm{rad}$.

The analytical theories of the motion of planets as well as the theories of the rotation of the Earth need a definition of the ecliptic or a plane close to the ecliptic. Just as the ICRF is a reference frame close to the equator J2000, a plane should be defined as a plane close to the mean ecliptic J2000, but not dependent on it. Its origin must be non-rotating. We choose for the origin the origin of the ICRF and we propose the definition of a conventional ecliptic as being defined from the ICRF by a rotation about the $x$ axis of $\varepsilon_{\text {conv }}$ where

$\varepsilon_{\mathrm{conv}}=0.409092614 \mathrm{rad}$ exactly.

Henceforth, the new planetary theories will be reported relative to the conventional ecliptic.

The angles $\psi, \omega$ and $\varphi$ which entirely define the rotation of the Earth will be used for $\psi$ in the conventional ecliptic from the origin of the ICRF, for $\omega$ from the conventional ecliptic and for $\varphi$ from the intersection of the true equator of date and the conventional ecliptic.

\section{About an alternative representation of the Earth Orientation Parameters (EOP)}

In the same manner as new expressions for precession were given in SMART (Sect. 2), a simplification of the implementations of the Earth Orientation Parameters (EOP) can be proposed for basic users. They describe the observed Earth variations of rotation, the observed offsets in the pole positions and polar motion by the means of the same Euler angles used in SMART. The plane of reference is a fixed plane, the conventional ecliptic, described in Sect. 3.

\subsection{Current EOP}

The EOP are the 5 Earth orientation parameters obtained by comparison of the current Earth rotation models and IERS observations. The parameters are the differences $(U T 1-U T C)$ or $(U T 1-T A I)$, the coordinates of the terrestrial pole and the celestial pole offsets (IERS Conventions, 1996).

The celestial pole offsets are observed variations of the celestial pole positions induced by mismodeling in precession and nutation theories in the International Celestial Reference Frame. Till the new 2000 IERS Conventions, these observed variations were given as differences in longitude and in obliquity referred to the equator and the equinox of date $(\mathrm{d} P, \mathrm{~d} \epsilon)$, (noted as ( $\mathrm{d} \psi, \mathrm{d} \epsilon$ ) by IERS) with respect to the conventional celestial pole coordinates defined by models (IERS Conventions, 1996). However, as it is recommended in the IERS Conventions (2000), such offsets between observed coordinates of the celestial pole $\left(X_{\mathrm{obs}}, Y_{\mathrm{obs}}\right)$ and computed ones must be provided as $(\delta \mathrm{X}, \delta \mathrm{Y})$ corrections where:

$\delta X=X_{\text {obs }}-\left(\zeta_{0}-d \alpha_{0} \bar{Y}+\bar{X}\right)$

$\delta Y=Y_{\mathrm{obs}}-\left(\eta_{0}+d \alpha_{0} \bar{X}+\bar{Y}\right)$

with $(\bar{X}, \bar{Y})$ being the coordinates of the pole derived from precession-nutation models (Capitaine 1990). The constants $\left(\zeta_{0}, \eta_{0}\right)$ are estimated by the precession-nutation series (for example, IAU2000A nutation series in IERS Conventions 2000) and $\mathrm{d} \alpha_{0}$ was estimated in using estimation of the lunar orbit fitted to LLR data (Chapront et al. 1999). 
After data analysis, IERS also provides the differences between $U T 1$ and $T A I$ or $U T 1$ and $U T C$. These differences describe the variations of the Earth rotation. $(U T 1-U T C)$ published by IERS is connected to the sideral time $S T$ (IERS Conventions, 1996) with:

$S T=K+r .((U T 1-U T C)+U T C)+P N_{\alpha}$

where $K=G M S T_{0 h U T 1}+L, L$ being the longitude of the site where the sideral time is computed. $P N_{\alpha}$ is precession and nutation terms in right ascension and $r$ is the ratio of universal to sideral time given by Aoki et al. (1982).

The last EOP are the coordinates of the celestial pole relative to the International Terrestrial Reference Frame (ITRF). After correcting for the celestial pole offsets and the variations of the Earth rotation, a residual rotation remains. It corresponds to pole position variations caused by free Chandler wobble and induced by atmospheric and oceanic mass redistribution. These variations, that have amplitudes of several tenths of arcseconds and quasidiurnal period in Euler angles, could also be seen as differences between the coordinates of the celestial pole in a rotating frame at the time of observation and the observed coordinates of the terrestrial pole in the ITRF. Therefore, they are associated with two small angles $(x, y)$ defined as the celestial pole coordinates relative to the International Terrestrial Reference Frame (ITRF).

\subsection{EOP as Euler angles}

To describe the EOP in Euler angles, we note $(\delta X, \delta Y)$ two angles of a small rotation in the equatorial J2000 frame. This rotation could also be written as small variations of Euler angles $(\psi, \omega)$.

$$
\begin{aligned}
\delta X= & \cos \omega \sin \psi \mathrm{d} \omega+\sin \omega \cos \psi \mathrm{d} \psi \\
\delta Y= & \left(\sin \epsilon_{0} \sin \omega-\cos \epsilon_{0} \cos \omega \cos \psi\right) \mathrm{d} \omega \\
& +\left(\cos \epsilon_{0} \sin \omega \sin \psi\right) \mathrm{d} \psi .
\end{aligned}
$$

We can then write the variations of the Euler angles versus $(\delta X, \delta Y)$ :

$\mathrm{d} \psi=\frac{1}{A}\left[\left(\sin \epsilon_{0}-\frac{\cos \epsilon_{0} \cos \psi \sin \omega}{\cos \omega}\right) \delta X-\frac{\sin \psi \sin \omega}{\cos \omega} \delta Y\right]$

$\mathrm{d} \omega=\frac{1}{A}\left[-\cos \epsilon_{0} \sin \psi \delta X+\cos \psi \delta Y\right]$

where $A=\cos \psi \sin \epsilon_{0} \sin \omega-\cos \epsilon_{0} \cos \omega$.

Furthermore, in Bretagnon et al. (1997), the angle of the Earth rotation is also given as an Euler angle $\varphi$, measured from the intersection of the equator of date and the ecliptic J2000. $\varphi$ is connected to the sideral time, $S T$, measured from the equinox of date, by the relation:

$\varphi=S T+\chi$

where $\chi$ is the arc between the origins (Fig. 1), and is function of the precession-nutation model. In order to use an Euler angles representation, it is convenient to consider the EOP values of $(U T 1-U T C)$ as variations of $\varphi$. One can then estimate a value of $\varphi$ taking into account the variations of the Earth rotation and one value without, and compute the difference $\mathrm{d} \varphi$.
Then, in using 11 , one obtains $\mathrm{d} \varphi=r .(U T 1-U T C) . \mathrm{d} \varphi$ represents the variations of the Euler angle for the Earth rotation induced by $(U T 1-U T C)$.

As it was described in the previous subsection, the EOP are published as small variations of the pole coordinates in the GCRF and ITRF and variations of angles of the Earth rotation. It is then possible to transform these small rotations in Euler angles in following the equations system 12 and 13 . We will then obtain 3 Euler angles representing the EOP, and a global rotation could then be estimated.

If we note $\left(\mathrm{d} \psi_{\mathrm{PO}}, \mathrm{d} \omega_{\mathrm{PO}}\right)$, the rotation induced by the pole offsets (in a non-rotating frame), $\left(\mathrm{d} \psi_{\mathrm{PM}}, \mathrm{d} \omega_{\mathrm{PM}}\right)$, the rotation from the polar motion (in a rotating frame) and $\delta S T$, the variation of the Earth rotation, then the global rotation induced by the variations of the reference pole coordinates in GCRF and ITRF and by the variations of the Earth rotation can be described by the Euler angles $\left(\mathrm{d} \psi_{\mathrm{G}}, \mathrm{d} \omega_{\mathrm{G}}, \mathrm{d} \varphi_{\mathrm{G}}\right)$ such as:

$$
\begin{aligned}
\mathrm{d} \psi_{\mathrm{G}} & =\mathrm{d} \psi_{\mathrm{PO}}+\mathrm{d} \psi_{\mathrm{PM}} \\
\mathrm{d} \omega_{\mathrm{G}} & =\mathrm{d} \omega_{\mathrm{PO}}+\mathrm{d} \omega_{\mathrm{PM}} \\
\mathrm{d} \varphi_{\mathrm{G}} & =r .(U T 1-U T C) .
\end{aligned}
$$

\subsection{Discussion}

As it was demonstrated and discussed by several authors (Capitaine 1990; Bizouard 1995), the main difficulty of the representation of the EOP as Euler angles is the commensurability of the observed periods used to estimate the EOP.

The polar coordinates variations or polar motion $\left(\mathrm{d} \psi_{\mathrm{PM}}\right.$, $\left.\mathrm{d} \omega_{\mathrm{PM}}\right)$ are detected from observations with periods smaller than 2 days, but the celestial pole offsets $\left(\mathrm{d} \psi_{\mathrm{PO}}, \mathrm{d} \omega_{\mathrm{PO}}\right)$ are estimated after analysis of multi-day observations. If we include in the Euler angles the polar motion and the pole offsets, then we will mix effects which have different frequency domains. Such questions are quite complex, but new results (Richter 2001) tend to demonstrate that the correlation problems seem to be solved in using very dense sets of observations. Nevertheless, in waiting for a more definitive conclusion, it is still possible, after the computation of the 5 classical EOP by comparison to observations, to publish for the basic users the EOP as three Euler angles. Such publication, containing Euler angles given with tabulated coefficients, will make the EOP easier to use for astronomers who are not specialists in Earth rotation and who do not want to do geophysical studies, but who want to reduce very accurate astrometric observations in order to obtain very precise orbits or positions of solar system objects, stars, quasars, etc. However, as it was stressed by Rothacher (2001), such tabulated publication would face the problem of the high number of coefficients needed to allow linear or quadratic interpolations. Studies must be lead to estimate the frequency at which the Euler angles would have to be estimated and the amount of data needed for the publications. The use of the nonrigid SMART solution associated with the representation of the EOP as Euler angles will give a consistent model for the Earth rotation in the GCRF and ITRF. 


\section{Considerations on the precession-nutation variables and on the arguments of the nutation}

\subsection{Precession-nutation variables}

Of course, precession and nutation are no longer separate and each variable $\psi, \omega$ and $\varphi$ contains a polynomial part (precession) and a periodic and Poisson part (nutation).

The variables $\theta, \zeta$ and $z$ defining the mean equator of date with respect to the equator $\mathrm{J} 2000$ cannot represent the precession-nutation because they are singular in the time origin. They are therefore useless.

\subsection{Arguments of the series of the nutation}

We note finally that a significant effort was accomplished to define a non-rotating origin, the origin of the ICRF, and angles reckoned from this origin such as the mean longitudes of the planets.

It is thus necessary to describe nutation as Fourier and Poisson series whose angles are linear combinations of 12 arguments. These arguments are the 8 mean longitudes of planets reckoned from the origin of the ICRF, the angles of Delaunay $D, F, l$ independent of the origin and the angle $\varphi$ of rotation of the Earth about its axis.

In any case, no argument must be reckoned from the equinox of date (Bretagnon 1998).

\section{Conclusion}

The polynomials of precession resulting from the precessionnutation SMART97 solution including the modification of precession constant given by (1) are gathered in Table 2 . These expressions of the precession are consistent with the IAU2000A nutation model adopted by IAU in 2000.

For the adopted value of the precession constant

$$
\begin{aligned}
\left(\frac{\mathrm{d} \mathcal{P}_{A}}{\mathrm{~d} t}\right)_{t=\mathrm{J} 2000.0} & =50287.92262^{\prime \prime} / \mathrm{tjy}, \\
\left(\frac{\mathrm{d} \psi_{A}}{\mathrm{~d} t}\right)_{t=\mathrm{J} 2000.0} & =-50384.78750^{\prime \prime} / \mathrm{tjy},
\end{aligned}
$$

these expressions ensure a precision of a few microarcseconds over the time interval (1900-2100).

Taking into account the adoption of the ICRF by IAU in 1997 , we propose to define a conventional ecliptic frame obtained from the ICRF by a rotation about the $x$ axis of $\varepsilon_{\mathrm{ICRF}}$ (Eq. (10)). This conventional ecliptic will be the reference frame in which the analytical theories of the motion of planets will be built. It is also with respect to this reference frame that the Euler's angles must be defined.

After the construction of a non-rigid SMART solution using the transfer function MHB2000 of Mathews et al., we propose to provide a precession-nutation solution expressed in the Euler variables and containing the diurnal and subdiurnal terms. We propose also an alternative representation of the Earth Orientation Parameters as corrections of the Euler's angles.

Acknowledgements. We wish to thank Dr. J. Vondrak for his useful comments and suggestions.

\section{References}

Bizouard, Ch. 1995, Thèse de doctorat, Observatoire de Paris

Bretagnon, P. 1998, in ed. J. Vondrak, \& N. Capitaine, Proceedings of the Journées 1997, Systèmes de référence spatio-temporels, Prague, 61

Bretagnon, P., Rocher, P., \& Simon, J.-L. 1997, A\&A, 319, 305

Bretagnon, P., Francou, G., Rocher, P., \& Simon, J.-L. 1998, A\&A, 329,329

Capitaine, N. 1990, Cel. Mec. Dyn. Astr., 48, 127

Capitaine, N. 2000, IAU Colloq. 180, Towards models and constants for sub-microarcseconds astrometry, ed. K. J. Johnston, D. D. McCarthy, \& G. H. Kaplan

Chapront, J., Chapront-Touzé, M., \& Francou, G. 1999, A\&A, 343, 624

IAU 1997, Proceedings of the Twenty-Three General Assembly, Kyoto, Japan

IAU 2000, Proceedings of the Twenty-Four General Assembly, Manchester, UK

Kinoshita, H. 1977, Cel. Mec., 15, 277

Lieske, J. H., Lederle, T., Fricke, W., \& Morando, B. 1977, A\&A, 58,1

Mathews, P. M., \& Herring, T. A. 2000, IAU Colloq. 180, Towards models and constants for sub-microarcseconds astrometry, ed. K. J. Johnston, D. D. McCarthy, \& G. H. Kaplan

Mathews, P. M., Herring, T. A., \& Buffet, B. A. 2002, J. Geophys. Res., in press

McCarthy, D. D. 1996, IERS Conventions (1996), IERS Technical Note 21, Observatoire de Paris

McCarthy, D. D. 2002, IERS Conventions (2000), IERS Technical Note, Observatoire de Paris, in press

Richter, B. 2001, IAG 2001 Scientific Assembly, Budapest, 2001 Feb. 8-9

Rothacher, M. 2002, IERS Workshop, Paris, 2002 Apr. 18-19

Simon, J. L., Bretagnon, P., Chapront, J., et al. 1994, A\&A, 282, 663

Souchay, J., Loysel, B., Kinoshita, H., \& Folgueira, M. 1999, A\&AS, 135,111

Williams, J. G. 1994, AJ, 108, 711 